\title{
Immunomagnetic B cell isolation as a tool to study blood cell subsets and enrich B cell transcripts
}

\author{
Amanda N. Henning ${ }^{1 *}$ (D), Daniel Green², Ryan Baumann ${ }^{1}$, Patrick Grandinetti ${ }^{3}$, Steven L. Highfill', \\ Huizhi Zhou ${ }^{1}$ and Valeria De Giorgi ${ }^{1}$
}

\begin{abstract}
Objective: Transcriptional profiling of immune cells is an indispensable tool in biomedical research; however, heterogenous sample types routinely used in transcriptomic studies may mask important cell type-specific transcriptional differences. Techniques to isolate desired cell types are used to overcome this limitation. We sought to evaluate the use of immunomagnetic B cell isolation on RNA quality and transcriptional output. Additionally, we aimed to develop a B cell gene signature representative of a freshly isolated B cell population to be used as a tool to verify isolation efficacy and to provide a transcriptional standard for evaluating maintenance or deviation from traditional B cell identity.

Results: We found RNA quality and RNA-sequencing output to be comparable between donor-matched PBMC, whole blood, and B cells following negative selection by immunomagnetic B cell isolation. Transcriptional analysis enabled the development of an 85 gene B cell signature. This signature effectively clustered isolated B cells from heterogeneous sample types in our study and naïve and memory B cells when applied to transcriptional data from a published source. Additionally, by identifying B cell signature genes whose functional role in B cells is currently unknown, our gene signature has uncovered areas for future investigation.
\end{abstract}

Keywords: RNA-sequencing, B cells, Differential gene expression, Gene signature

\section{Introduction}

The rise of increasingly affordable and accessible nextgeneration sequencing (NGS) technologies has made transcriptional profiling via RNA-sequencing (RNAseq) an achievable and essential research tool [1]. Whole blood (WB) and peripheral blood mononuclear cells (PBMC) are routinely used sample types in RNA-seq studies due to their ease of collection and capacity for long-term storage. However, while they provide a wealth of biological information, the heterogeneity of these sample types can be a drawback, as cell type-specific

\footnotetext{
*Correspondence: amanda.henning@nih.gov

${ }^{1}$ Department of Transfusion Medicine, Clinical Center, National Institutes

of Health, Bethesda, MD, USA

Full list of author information is available at the end of the article
}

transcriptional differences can be masked in bulk RNAseq approaches [2].

When transcriptional information on a discrete cell type is required, multiple experimental techniques are available to overcome sample heterogeneity, including single cell sequencing and fluorescence-activated cell sorting (FACS). While these techniques are highly effective, they require specialized equipment and can be costly [3]. The most feasible approach for many labs is the use of immunomagnetic separation methods, as it is cost-effective, requires minimal specialized training or equipment, and many commercially available kits exist for isolating standard immune cell components [4].

$\mathrm{B}$ cells are an immune cell type of particular interest in biomedical research, representing a cornerstone of adaptive immunity, with direct involvement in certain 
cancers and autoimmune disorders [5, 6]. There have been a number of studies identifying B cell gene expression patterns associated with various disease states, including non-Hodgkin's lymphoma [7] and autoimmune diseases [8]. Fewer studies have attempted to identify a $B$ cell gene signature from healthy donors relative to a heterogenous population $[9,10]$, and most of these studies were performed using microarrays, which can be limited in their sensitivity and reproducibility [11]. With the increasing importance of subset-specific transcriptional analysis in disease research, having a high-quality B cell gene signature obtained via modern NGS technologies is critical. In this study, we have evaluated the RNA quality and sequencing output of B cells isolated using negative selection immunomagnetic cell separation and have established a baseline B cell gene signature from healthy donors. Our B cell signature provides a useful tool for verifying $B$ cell purity at the transcriptional level and establishes a transcriptional baseline for assessing deviations incurred by environmental or experimental perturbations. Furthermore, our signature has identified genes that play an unknown role in B cell function that are of interest for future investigations.

\section{Main text \\ Methods \\ Sample collection, processing, and NGS}

Human whole blood samples were obtained from healthy donors on an IRB-approved NIH protocol (99-CC-0168). PBMC were isolated from whole blood using FicollPaque Plus solution (GE Healthcare), and B cells were subsequently isolated using the EasySep ${ }^{\mathrm{TM}}$ Human B cell Isolation kit (StemCell Technologies). RNA was isolated from $200 \mu \mathrm{L}$ whole blood using the Quick-RNA Whole Blood kit (Zymo Research), and RNA was isolated from PBMC and B cells using the RNeasy Plus Mini kit (Qiagen). RNA was assessed for quality on an Agilent 2100 Bioanalyzer (Agilent Technologies). Library preparations were done using TruSeq Stranded mRNA Library Prep (Illumina). RNA input was $300 \mathrm{ng}$ for $\mathrm{WB}$ and PBMC samples and $100 \mathrm{ng}$ for isolated B cells. Libraries were normalized to $10 \mathrm{nM}$, and equal volumes of all 12 libraries were pooled together for sequencing on a NextSeq 550 instrument (Illumina).

\section{Bioinformatic and statistical analysis}

Sequenced reads were aligned to the human reference genome (UCSC hg19) using the RNA-Seq Alignment application (v2.0.1) on the BaseSpace Sequencing Hub (Illumina). Sequencing files are available on the GEO repository: GSE186768. Differential expression analysis was performed in R (v4.0.2) [12] using the DESeq2 package (v1.28.1). Genes were considered to be differentially expressed if they had a $\log 2$ fold change of $<-1$ or $>1$ and an adjusted p-value (padj) of $<0.05$. Bioinformatic analyses were performed using the topGO R package (v2.40.0), the web-based WebGestalt analysis tool (www.webge stalt.org) [13], and the GSEA (v4.0.3) desktop application $[14,15]$. For validation of our B cell gene signature, the RNA-seq dataset from Monaco et al. [16] was downloaded from GEO: GSE107011. The ImmGen database (https://www.immgen.org/) was used to investigate signature gene expression levels. Additional statistics and figure creation were done using GraphPad Prism (v8.4.3; GraphPad Software). For a more detailed methodological description, see Additional file 1.

\section{Results and discussion}

\section{RNA quality and NGS output is consistent among WB, PBMC} and isolated $B$ cells

To compare the quality of RNA and sequencing data generated from related sample types, we collected donormatched WB and PBMC from four healthy donors (HD). Immunophenotyping of PBMC revealed some inter-donor variability (Fig. 1a, b; Additional file 2: Fig. S1a); however, the $\mathrm{CD} 19^{+} \mathrm{B}$ cell population was consistent at $7 \%$. B cells were then isolated from fresh PBMC using the StemCell ${ }^{\mathrm{TM}}$ EasySep $^{\mathrm{TM}}$ Human B cell isolation kit. This kit utilizes negative selection to isolate B cells. In this way, non-B cells are labeled with antibodies conjugated to magnetic particles, and the cells remaining after magnetic separation constitute an enriched B cell population. B cell purity was assessed via flow cytometry in all samples, and we observed a robust enrichment of B cells, with $>98 \%$ of $\mathrm{CD} 45^{+}$lymphocytes expressing CD19 (Fig. 1a; Additional file 2: Fig. S1b). RNA was isolated from WB, PBMC and B cells, and RNA quality was assessed based on RNA integrity number (RIN). All samples demonstrated high RIN scores (RIN $>8$ ) suitable for NGS studies, and yielded sufficient RNA for NGS library preparation (Table 1).

Samples were sequenced on the Illumina NextSeq550 platform. The total number of reads/samples, percent of reads mapping to abundant regions of the genome, percent aligned reads, and fold coverage across coding regions were relatively consistent across different sample types (Table 1). Overall, negative selection B cell isolation was highly efficient and resulted in RNA and sequencing output that was of similar quality among sample types. In this way, the additional processing steps required for $B$ cell isolation did not adversely affect experimental results.

\section{Identification of $a$ B cell gene signature}

RNA-sequencing was performed on freshly isolated, donor-matched WB, PBMC, and B cells (Additional 
A
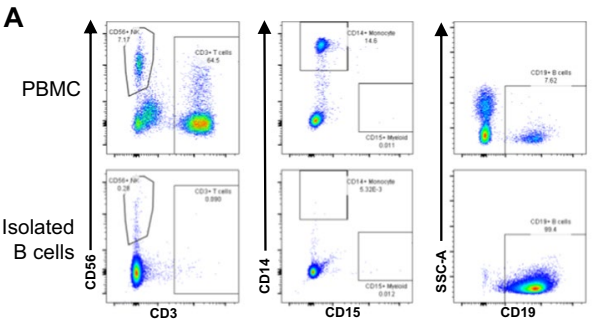

B

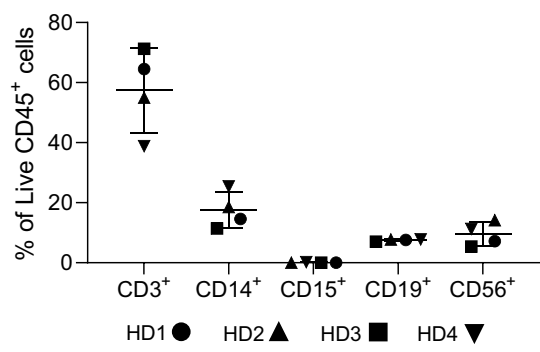

C

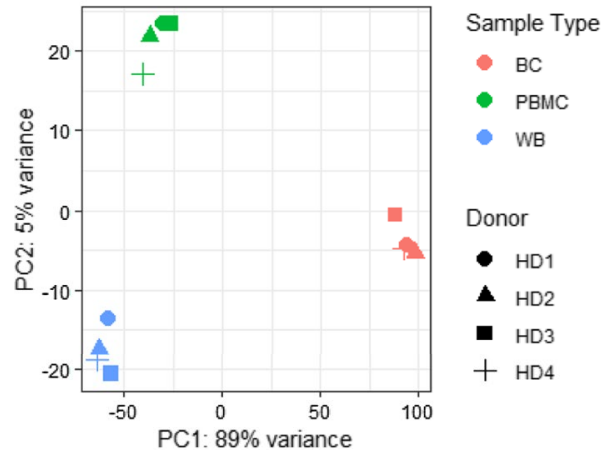

E

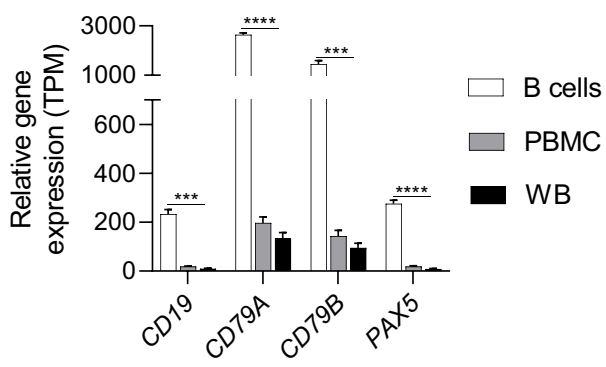

$\mathbf{F}$

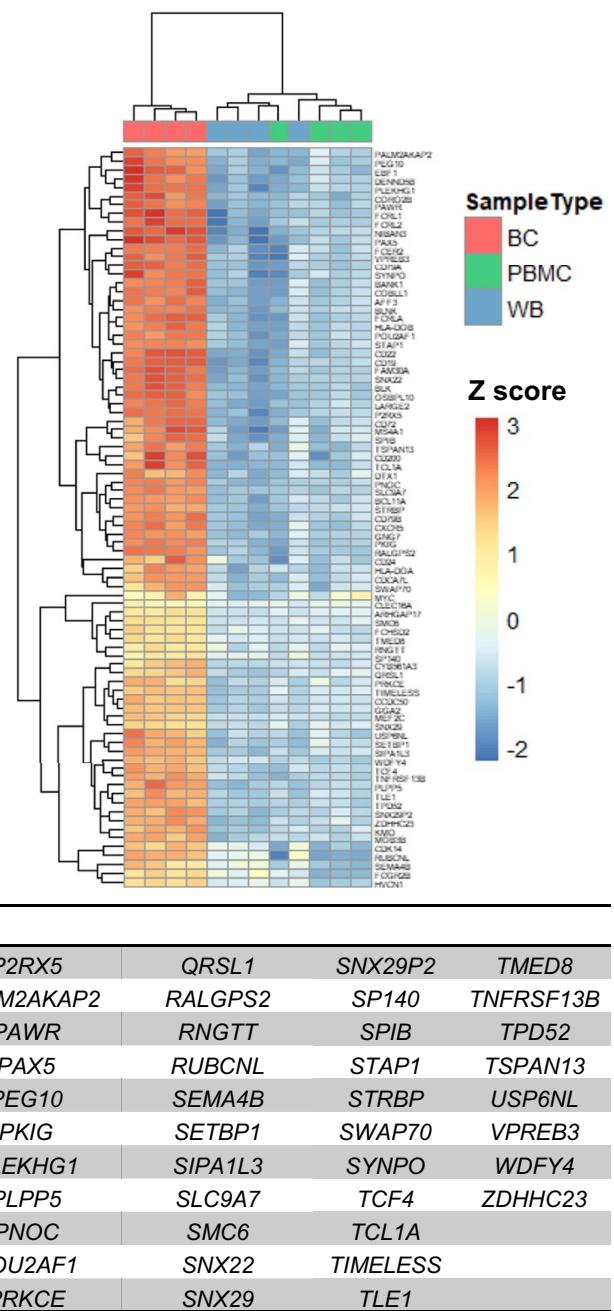

Fig. 1 Identification of a B cell gene signature. a Representative flow plots of PBMC immunophenotyping (top) and B cell purity check (bottom). Cells were previously gated on Lymphocytes/Single cells/Live/CD45+. b Summary of immunophenotyping results for PBMC samples. Individual values are displayed along with the average \pm SD. c PCA plot from transcriptional analysis of whole blood (WB), PBMC and isolated B cells (BC). $\mathbf{d}$ List of 85 genes that make up the B cell gene signature. e Relative gene expression of select B cell signature genes. Statistics computed using unpaired $T$ tests with correction for multiple comparisons. Graph displays average \pm SD. $p$-value applies to comparisons of $B$ cells versus both PBMC and WB. f. Heatmap with hierarchical clustering using the B cell gene signature. ${ }^{*} p<0.05 ;{ }^{* *} p<0.01{ }^{* * *} p<0.001 ;{ }^{* * *} p<0.0001$

file 3: Table S1). Principle component analysis (PCA) demonstrated tight and distinct clustering of sample types, irrespective of donor (Fig. 1c), and differential expression analysis identified 7027 differentially expressed genes (DEGs) between $B$ cells and WB and 5,537 DEGs between B cells and PBMC (Additional file 3: Table S2). Significant gene ontology (GO) terms related to $B$ cell-specific functions were identified, and gene set 
Table 1 RNA quality and NGS output among sample types

\begin{tabular}{lccc}
\hline RNA quality and yield & Whole blood & PBMC & Isolated B cells \\
\hline RIN & $9.28(0.3)$ & $9.75(0.3)$ & $9.78(0.2)$ \\
RNA yield (ng) & $301(30.9)$ & $559(364.2)$ & $186(137.4)$ \\
$\begin{array}{l}\text { Sequencing output } \\
\quad \text { Total reads }\left(\times 10^{6}\right)\end{array}$ & $53.97(7.9)$ & $47.21(2.45)$ & $57.31(4.86)$ \\
$\begin{array}{l}\text { Abundant reads (\% } \\
\text { of total) }\end{array}$ & $7.60(1.33)$ & $7.46(1.70)$ & $9.87(1.19)$ \\
$\begin{array}{l}\text { Aligned reads (\% } \\
\text { filtered) }\end{array}$ & $97.60(0.33)$ & $97.88(0.28)$ & $94.93(1.74)$ \\
$\begin{array}{l}\text { Fold coverage (cod- } \\
\text { ing) }\end{array}$ & $113.32(20.53)$ & $103.92(6.54)$ & $100.69(11.92)$ \\
\hline
\end{tabular}

Values represent mean (SD), $n=4$ /group. RNA yield for whole blood corresponds to $n g / 200 \mu l$, and for PBMC and B cells yield represents ng/1e6 cells. RIN, RNA integrity number

enrichment analysis (GSEA) showed positive enrichment of B cell-specific gene sets (Additional file 2: Fig. S2a, b; Additional file 3: Table S3). Bioinformatic analyses, therefore, supported efficient B cell isolation and transcriptional capture.

Differential gene expression analysis in PBMC and B cell samples was used to identify a robust B cell gene signature indicative of a homogenous population derived from healthy individuals. The top 200 most significant DEGs between B cells and PBMC effectively clustered all samples and included 51 upregulated genes and 149 downregulated genes (Additional file 2: Fig. S2c). To expand our signature gene set, these 51 upregulated genes were combined with a B cell transcriptional module [17] to create a $B$ cell gene signature consisting of 85 genes upregulated in $\mathrm{B}$ cells relative to heterogenous PBMC samples (Fig. 1d). The module from Chaussabel et al. [17] was derived using computationally identified patterns of coordinately expressed genes in PBMC microarrays sourced from multiple disease states. We felt that combining gene sets identified using these two divergent methodological approaches would create a robust B cell gene signature. Indeed, this gene set was highly enriched for B cell-specific GO terms (Additional file 2: Fig. S3a), included a number of highly expressed genes with critical B cell functions (Fig. 1e; Additional file 2: Fig S3b), and resulted in distinct clustering of B cell samples relative to heterogenous sample types (Fig. 1f).

\section{Validation of B cell gene signature in external dataset}

To validate our B cell gene signature, we used transcriptional data published by Monaco and colleagues [16] consisting of RNA-seq data from sorted immune cell types, including multiple B cell subsets. B cell subsets were isolated via FACS and were classified as: naïve $\left(\mathrm{CD} 27^{-} \mathrm{IgD}^{+}\right)$, non-switched memory $\left(\mathrm{CD} 27^{+} \mathrm{IgD}^{+}\right)$, switched memory $\left(\mathrm{CD}_{2} 7^{+} \mathrm{IgD}^{-}\right)$, exhausted memory or double negative $(\mathrm{DN})\left(\mathrm{CD}^{-} 7^{-} \mathrm{IgD}^{-}\right)$, and plasmablasts $\left(\mathrm{CD} 27^{+} \mathrm{IgD}^{-} \mathrm{CD} 38^{\mathrm{hi}}\right)$. As the immunomagnetic separation method we used did not discriminate between specific B cell subsets, we wanted to ensure that our B cell signature was indicative of these multiple B cell developmental states. Hierarchical clustering using our B cell gene signature was performed on log2-transformed Transcripts Per Million (TMP) data. Our B cell signature effectively clustered naïve and memory B cell populations from heterogenous PBMC samples (Fig. 2); however, plasmablasts demonstrated a divergent gene expression profile. This is to be expected since, compared to naïve and memory B cell, plasmablasts are a small component of the circulating B cell population (96\% vs 1\%) [18], and thus contributed little to our B cell signature. Furthermore, plasmablasts, along with plasma cells, represent a highly specialized $B$ cell subset with a unique transcriptional profile. They have been shown to downregulate a number of traditional $B$ cell genes, including CD24, CXCR5, PAX5, EBF1, and SPIB [19], all of which were part of a downregulated cluster (Fig. 2) in our analysis. Since some genes crucial for B cell function can also be expressed in other immune cell types, we wanted to verify that our B cell gene signature would robustly identify B cells when challenged with other homogenous non-B cell populations. Hierarchical clustering was performed on the same external dataset [16], this time using sorted B cell subsets, heterogenous PBMC samples, and 24 additional sorted leukocyte subsets. Once again, our $B$ cell signature effectively clustered naïve and memory B cell populations separate from all other leukocyte cell types (Additional file 2: Fig. S4). Overall, a validation of our B cell gene signature using an external dataset found it to be indicative of naïve and memory B cell subsets relative to both a heterogenous PBMC population as well as homogenous non-B cell leukocyte populations.

\section{Manual characterization of B cell signature genes}

An in-depth exploration of $B$ cell signature genes revealed that over half have known functional roles in $\mathrm{B}$ cells (Additional file 2: Fig. S5). The remaining genes had no reported function in B cells; however, they represent promising areas of further investigation, as their significant expression in B cells suggests involvement in functional pathways. Indeed, CCDC50, KMO, PAWR, PEG10, and PLPP5 may play a role in multiple $B$ cell-associated cancers [20-24], and CDCA7L and OSBPL10 may be risk markers in multiple myeloma and diffuse large B cell lymphoma, respectively [25, 26]. Additionally, investigation of B cell signature gene expression levels via the Immunological Genome Project (https://www.immgen.org/) Human Expression 


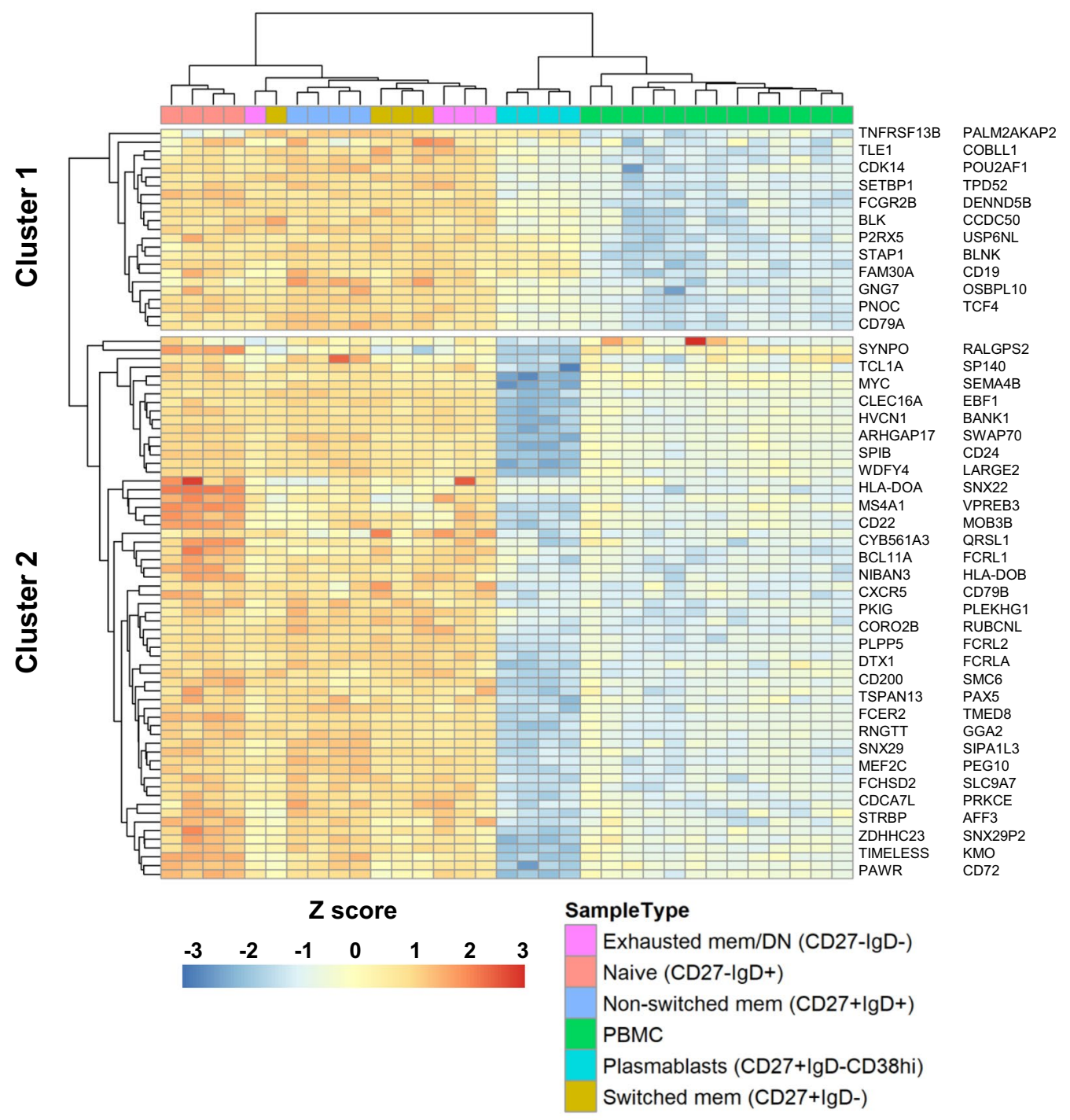

Fig. 2 Validation of B cell gene signature. Heatmap with hierarchical clustering using the B cell gene signature and RNA-seq data from Monaco et al. (16). B cell subsets are indicated at the top, and cell surface markers used for sorting are listed in the legend. Genes corresponding to each cluster are listed on the side, and gene order corresponds to their order in the heatmap. DN, double negative

Data identified 39 genes as having B cell-specific gene expression (Additional file 2: Fig. S5, Additional file 3: Table S4). This included expected genes, such as many involved in the BCR signaling pathway, but also genes of unknown function in B cells, including PLEKHG1, RALGPS2, and SYNPO, among others. We have thus identified a $B$ cell gene signature representative of a freshly isolated, homogenous B cell population consisting of both well-characterized B cell genes and novel genes whose functional characterization may provide insight in the understanding of B cell malignancies.

\section{Conclusions}

RNA-sequencing performed in donor matched WB, PBMC, and isolated $B$ cells has verified the use of negative selection immunomagnetic cell separation as a viable way to isolate B cells for NGS studies. We have identified a B cell gene signature representative of a freshly isolated, homogenous B cell population. In particular, our signature may be used for transcriptional verification of naïve or memory B cell identity, especially in instances where immunophenotyping is not possible, or to assess deviation from the traditional B cell transcriptome following 
chemical or genetic perturbation. Our B cell gene signature consists of many genes with well-characterized roles in B cell development and function; however, the identification of many genes with unknown B cell functions represents an important area for future investigations to enhance our understanding of B cell-related malignancies.

\section{Limitations}

The limitations of this study include the relatively small sample size and our lack of B cell subset composition information for isolated samples. Additionally, we utilized a negative selection kit so as to avoid inadvertent B cell activation; however, it would be beneficial to experimentally verify the transcriptional effect of negative enrichment kits relative to positive enrichment kits or other forms of B cell isolation.

\section{Abbreviations}

DEGs: Differentially expressed genes; DN: Double negative; FACS: Fluorescence-activated cell sorting; GO: Gene ontology; GSEA: Gene set enrichment analysis; HD: Healthy donor; NGS: Next-generation sequencing; PBMC: Peripheral blood mononuclear cells; RIN: RNA integrity number; RNA-seq: RNA sequencing; TPM: Transcripts per million; WB: Whole blood.

\section{Supplementary Information}

The online version contains supplementary material available at https://doi. org/10.1186/s13104-021-05833-z.

\section{Additional file 1. Extended methods.}

Additional file 2: Fig. S1. Immunophenotyping of PBMC and isolated B cells. Fig. S2. Transcriptional analysis of donor-matched WB, PBMC, and isolated B cells. Fig. S3. Investigating the B cell gene signature. Fig. S4. Validation of B cell gene signature. Fig S5. Functional role of B cell signature genes.

Additional file 3: Table S1. TPM values across Sample Types. Table S2. Lists of differentially expressed genes between sample types. Table S3. Significantly enriched GO terms ( $p$-value $<0.01)$ from upregulated DEGs in freshly isolated B cells vs PBMC and WB. Table S4. Investigating expression levels of $B$ cell signature genes.

Acknowledgements

The authors thank NIH blood donors for their participation in the study.

\section{Authors' contributions}

ANH performed data analysis, generated all paper figures and wrote the manuscript. VDG conceived, designed, supervised the research study and critically reviewed the manuscript; SH, DG, RB, PG, HZ supported the experimental work; SH, DG critically reviewed the manuscript. All authors read and approved the final manuscript.

\section{Funding}

Open Access funding provided by the National Institutes of Health $(\mathrm{NIH})$. This work was supported by the Intramural Research Program of the NIH Clinical Center (project Z99 CL999999).

\section{Availability of data and materials}

The datasets generated and analyzed in the current study are available in the Gene Expression Omnibus (GEO) repository. RNA-seq data generated in this study can be found at GEO accession: GSE186768, and previously published data analyzed in this study can be found at GEO accession: GSE107011.

\section{Declarations}

\section{Ethics approval and consent to participate}

Samples for this study were obtained from healthy donors as part of a National Institutes of Health Clinical Center institutional review board-approved protocol (protocol 99-CC-0168; Clinical Trials Registration, NCT00001846). Donors provided written informed consent, and blood samples were de-identified prior to distribution.

\section{Consent for publication}

Not applicable.

\section{Competing interests}

The authors declare that they have no competing interests. The views expressed are the authors' own and do not represent the National Institutes of Health, the Department of Health and Human Services, or the U.S. Federal government.

\section{Author details}

${ }^{1}$ Department of Transfusion Medicine, Clinical Center, National Institutes of Health, Bethesda, MD, USA. ${ }^{2}$ Women's Malignancies Branch, National Cancer Institute, National Institutes of Health, Bethesda, MD, USA. ${ }^{3}$ Experimental Transplantation and Immunology Branch, National Cancer Institute, National Institutes of Health, Bethesda, MD, USA.

Received: 2 September 2021 Accepted: 3 November 2021

Published online: 18 November 2021

\section{References}

1. Casamassimi A, Federico A, Rienzo M, Esposito S, Ciccodicola A. Transcriptome Profiling in Human Diseases: New Advances and Perspectives. Int J Mol Sci. 2017;18:8.

2. Cobb JP, Mindrinos MN, Miller-Graziano C, Calvano SE, Baker HV, Xiao W, et al. Application of genome-wide expression analysis to human health and disease. Proc Natl Acad Sci U S A. 2005;102(13):4801-6.

3. Papalexi E, Satija R. Single-cell RNA sequencing to explore immune cell heterogeneity. Nat Rev Immunol. 2018;18(1):35-45.

4. Plouffe BD, Murthy SK, Lewis LH. Fundamentals and application of mag netic particles in cell isolation and enrichment: a review. Rep Prog Phys. 2015;78(1):016601.

5. Carter RH. B cells in health and disease. Mayo Clin Proc. 2006;81(3):377-84.

6. Shaffer AL, Rosenwald A, Staudt LM. Lymphoid malignancies: the dark side of B-cell differentiation. Nat Rev Immunol. 2002;2(12):920-32.

7. Staudt LM, Dave S. The biology of human lymphoid malignancies revealed by gene expression profiling. Adv Immunol. 2005;87:163-208.

8. Garaud JC, Schickel JN, Blaison G, Knapp AM, Dembele D, Ruer-Laventie J, et al. B cell signature during inactive systemic lupus is heterogeneous: toward a biological dissection of lupus. PLOS ONE. 2011;6(8):e23900.

9. Palmer C, Diehn M, Alizadeh AA, Brown PO. Cell-type specific gene expression profiles of leukocytes in human peripheral blood. BMC Genomics. 2006:7:115.

10. Abbas AR, Baldwin D, Ma Y, Ouyang W, Gurney A, Martin F, et al. Immune response in silico (IRIS): immune-specific genes identified from a compendium of microarray expression data. Genes Immun. 2005;6(4):319-31.

11. Draghici S, Khatri P, Eklund AC, Szallasi Z. Reliability and reproducibility issues in DNA microarray measurements. Trends Genet. 2006;22(2):101-9.

12. Team RC. R: A Language and Environment for Statistical Computing. 4.0.2 ed. Vienna, Austria: R Foundation for Statistical Computing; 2020.

13. Wang J, Duncan D, Shi Z, Zhang B. WEB-based GEne SeT AnaLysis Toolkit (WebGestalt): update 2013. Nucleic Acids Res. 2013;41(Web Server issue):W77-83.

14. Mootha VK, Lindgren CM, Eriksson KF, Subramanian A, Sihag S, Lehar J, et al. PGC-1alpha-responsive genes involved in oxidative 
phosphorylation are coordinately downregulated in human diabetes. Nat Genet. 2003;34(3):267-73.

15. Subramanian A, Tamayo P, Mootha VK, Mukherjee S, Ebert BL, Gillette MA, et al. Gene set enrichment analysis: a knowledge-based approach for interpreting genome-wide expression profiles. Proc Natl Acad Sci U S A. 2005;102(43):15545-50.

16. Monaco G, Lee B, Xu W, Mustafah S, Hwang YY, Carre C, et al. RNA-Seq Signatures Normalized by mRNA Abundance Allow Absolute Deconvolution of Human Immune Cell Types. Cell Rep. 2019:26(6):1627-40.

17. Chaussabel D, Quinn C, Shen J, Patel P, Glaser C, Baldwin N, et al. A modular analysis framework for blood genomics studies: application to systemic lupus erythematosus. Immunity. 2008;29(1):150-64.

18. Morbach H, Eichhorn EM, Liese JG, Girschick HJ. Reference values for B cell subpopulations from infancy to adulthood. Clin Exp Immunol. 2010;162(2):271-9.

19. Kassambara A, Reme T, Jourdan M, Fest T, Hose D, Tarte K, et al. GenomicScape: an easy-to-use web tool for gene expression data analysis. Application to investigate the molecular events in the differentiation of $B$ cells into plasma cells. PLoS Comput Biol. 2015;11(1):e1004077.

20. Trezise S, Karnowski A, Fedele PL, Mithraprabhu S, Liao Y, D'Costa K, et al. Mining the Plasma Cell Transcriptome for Novel Cell Surface Proteins. Int J Mol Sci. 2018;19:8.

21. Schleiss C, Carapito R, Fornecker LM, Muller L, Paul N, Tahar O, et al. Temporal multiomic modeling reveals a B-cell receptor proliferative program in chronic lymphocytic leukemia. Leukemia. 2021;35(5):1463-74.
22. Hu C, Xiong J, Zhang L, Huang B, Zhang Q, Li Q, et al. PEG10 activation by co-stimulation of CXCR5 and CCR7 essentially contributes to resistance to apoptosis in CD19+CD34+ B cells from patients with B cell lineage acute and chronic lymphocytic leukemia. Cell Mol Immunol. 2004;1 (4):280-94.

23. Greene JT, Mani R, Ramaswamy R, Frissora F, Yano M, Zapolnik K, et al. Par-4 overexpression impedes leukemogenesis in the Emicro-TCL1 leukemia model through downregulation of NF-kappaB signaling. Blood Adv. 2019;3(8):1255-66.

24. Farfsing A, Engel F, Seiffert M, Hartmann E, Ott G, Rosenwald A, et al. Gene knockdown studies revealed CCDC50 as a candidate gene in mantle cell lymphoma and chronic lymphocytic leukemia. Leukemia. 2009;23(11):2018-26.

25. Weinhold N, Meissner T, Johnson DC, Seckinger A, Moreaux J, Forsti A, et al. The 7p15.3 (rs4487645) association for multiple myeloma shows strong allele-specific regulation of the MYC-interacting gene CDCA7L in malignant plasma cells. Haematologica. 2015;100(3):e110.

26. Dobashi A, Togashi Y, Tanaka N, Yokoyama M, Tsuyama N, Baba S, et al. TP53 and OSBPL10 alterations in diffuse large B-cell lymphoma: prognostic markers identified via exome analysis of cases with extreme prognosis. Oncotarget. 2018;9(28):19555-68.

\section{Publisher's Note}

Springer Nature remains neutral with regard to jurisdictional claims in published maps and institutional affiliations.
Ready to submit your research? Choose BMC and benefit from:

- fast, convenient online submission

- thorough peer review by experienced researchers in your field

- rapid publication on acceptance

- support for research data, including large and complex data types

- gold Open Access which fosters wider collaboration and increased citations

- maximum visibility for your research: over 100M website views per year

At BMC, research is always in progress.

Learn more biomedcentral.com/submissions 